\title{
ENTEROBACTERIACEAE AND PSEUDOMONADACEAE ON THE DORSUM OF THE HUMAN TONGUE
}

\author{
Simone CONTI ${ }^{1}$, Silvana Soléo Ferreira dos SANTOS ${ }^{2}$, Cristiane Yumi KOGA-ITO ${ }^{3}$, Antonio Olavo Cardoso JORGE ${ }^{4}$
}

\author{
1- DDS, MSc student, Postgraduate Program in Dentistry, University of Taubaté, Taubaté, SP, Brazil. \\ 2- DDS, MSc, PhD, Assistant Professor of Microbiology and Immunology, Department of Dentistry, University of Taubaté, Taubaté, SP, Brazil. \\ 3- DDS, MSc, PhD, Assistant Professor of Microbiology and Immunology, São José dos Campos Dental School, São Paulo State University, São José \\ dos Campos, SP, Brazil. \\ 4- DDS, MSc, PhD, Chair Professor of Microbiology and Immunology, São José dos Campos Dental School, São Paulo State University, São José dos \\ Campos, SP, Brazil.
}

Corresponding address: Silvana Soléo Ferreira dos Santos - Rua Victor Barbosa Guisard, 35 - Centro - 12010-660 Taubaté, SP - Brasil - Phone: +55-123635-3466 - e-mail: silvana.soleo@uol.com.br

Received: July 4, 2008 - Modification: August 31, 2008 - Accepted: October 7, 2008

\begin{abstract}
$O$ bjective: The aim of this study was to correlate the presence of Enterobacteriaceae, Pseudomonadaceae, Moraxellaceae and Xanthomonadaceae on the posterior dorsum of the human tongue with the presence of tongue coating, gender, age, smoking habit and denture use. Material and Methods: Bacteria were isolated from the posterior tongue dorsum of 100 individuals in MacConkey agar medium and were identified by the API 20E system (Biolab-Mérieux). Results: $43 \%$ of the individuals, presented the target microorganisms on the tongue dorsum, with greater prevalence among individuals between 40 and 50 years of age $(p=0.001)$ and non-smokers ( $\mathrm{p}=0.0485)$. Conclusions: A higher prevalence of Enterobacteriaceae and Pseudomonadaceae was observed on the tongue dorsum of the individuals evaluated. There was no correlation between these species and the presence and thickness of tongue coating, gender and presence of dentures.
\end{abstract}

Key words: Enterobacteriaceae. Pseudomonadaceae. Oral cavity. Tongue.

\section{INTRODUCTION}

The microbial composition of dental biofilm has been extensively studied. However, fewer studies have evaluated the microbiota of the oral soft tissue which comprises at least $80 \%$ of the surfaces that can be colonized within the mouth ${ }^{10}$. Although the tongue dorsum appears to shelter a complex microbiological niche, knowledge of the role of tongue microbiota in systemic and oral diseases is very limited.

There has been increased interest in the study of the microbiota of tongue, due to its possible association with oral halitosis ${ }^{7,14,24}$. Previous studies have suggested that Enterobacteriaceae may be involved in halitosis, as they produce putrefied gases when they metabolize proteins or certain amino acids ${ }^{5}$. Nasal and paranasal secretion may accumulate in the posterior region of the tongue dorsum, favoring the formation of tongue coating causing halitosis as a result of amino acid degradation by bacteria under anaerobic conditions ${ }^{15}$.

The importance of tongue as a potential microbial reservoir has been suggested by some studies. A study of the ultrastructure of the morphotypes of bacteria residing to the tongue's surface showed the presence of 35 different bacterial morphotyes ${ }^{1}$. Tanner, et al. ${ }^{25}$, by using checkerboard DNA probe assay, verified that some cariogenic or periodontal pathogens microorganisms were more frequently isolated from tongue than tooth samples in children under 18 months, and suggested that the tongue is an important microbial reservoir. This role was also reported among adults and previous studies revealed that tongue might have a role in the recolonization of dental surfaces and be a reservoir of periodontal pathogens ${ }^{3}$.

Microorganisms of the Enterobacteriaceae family and Pseudomonas genus may act as opportunist pathogens and colonize the human oral cavity, especially in patients with debilitating diseases who are submitted to prolonged treatments with antibiotics or cytotoxic medications ${ }^{17,18,20,22}$. According to $\mathrm{Zhu}$, et al. ${ }^{27}$ the oral reservoir of coliforms in people suffering from stroke is noteworthy as K. pneumoniae may cause aspiration pneumonia.

Previous studies reported the presence of this group of microorganisms in the oral cavity. A clinical study conducted by Goldberg, et al. ${ }^{5}$ evaluated the prevalence of Enterobacteriaceae in four different populations and they detected Enterobacteriaceae in $48 \%$ of patients with complete dentures, $27.1 \%$ of patients with halitosis, $16.4 \%$ of controls and $13 \%$ of orthodontic patients. The species 
Klebsiella oxytoca, Klebsiella pneumoniae pneumoniae, Enterobacter gergoviae and Enterobacter cloacae were isolated from the tongue.

Increased counts of coliforms were observed among individuals with chronic nail-biting habit ${ }^{2}$ and after the installation of fixed orthodontic appliances ${ }^{6}$. On the other hand, no differences in oral carriage of coliforms were observed among stroke sufferers ${ }^{27}$ and HIV-infected patients ${ }^{26}$. During chemotherapy, Napeñas, et al. ${ }^{12}$ reported that the most frequent Gram-negative species isolated were from the Enterobacteriaceae family, Pseudomonas spp. and E. coli.

Considering the importance of the oral cavity as a reservoir of potentially pathogenic microorganisms, the aim of this study was to analyze the presence of bacteria of the Enterobacteriaceae, Pseudomonadaceae, Moraxellaceae and Xanthomonadaceae families in the posterior region of the tongue dorsum in a population of adult individuals. Also, the study aimed to correlate of the prevalence of these microorganisms with the presence of tongue coating, gender, age, smoking habit and denture use.

\section{MATERIAL AND METHODS}

The research protocol was approved by the local Research Ethics Committee (UNITAU/Protocol 163/05) and the volunteers signed an informed consent form prior to entering the study. One hundred individuals of both genders (50 females and 50 males) between 30 and 50 years of age were selected and evaluated with regard to the presence of tongue coating and dentures. The smoking habit, period of time from last oral hygiene, tongue cleaning habit, and whether the volunteer was capable to identify him/herself as having halitosis was evaluated by means of a questionnaire. Individuals who wore orthodontic appliances and used antibiotics or oral rinses within 6 months prior to the study were excluded.

The presence of coating on the tongue dorsum was quantified accordance with Tanaka, et al. ${ }^{24}$. The following scores were attributed to the tongue coating area: 0 ) none; 1) coating on the posterior third;2) coating on the posterior and middle thirds; and 3) coating on the posterior, middle, and anterior thirds of the tongue dorsum. Tongue coating thickness was defined by the following scores: 0 ) none; 1) thin layer with visible papillae; 2) moderate layer with some visible papillae; and 3) thick layer with no visible papillae.
Tongue coating was evaluated by a single calibrated examiner, after the percentage of agreement had been assessed. Initially, the area and thickness of tongue coating in 10 volunteers were measured, and after $24 \mathrm{~h}$, the same volunteers were evaluated again $(\mathrm{k}=0.9)$.

Material was collected from the tongue dorsum in the region of the circumvallated papillae with a calibrated sterile disposable $10 \mu \mathrm{L}$ loop (Inlab Diagnostic; Diadema, SP, Brazil). Sampling consisted in collecting material by scrapping the entire region until loop content was completed. Then, the material was transferred to safe-lock micro test tubes (Eppendorf, Barkhausenweg, Hamburg, Germany) containing $1 \mathrm{ml}$ of sterile PBS (phosphate buffered saline; $\mathrm{pH}$ 7.4), and homogenized for $30 \mathrm{~s}$ (Tube agitator, AP 56, Phoenix, Araraquara, SP, Brazil). An aliquot of $0.1 \mathrm{~mL}$ of this suspension was plated on MacConkey agar media plates (Difco, Detroit, MI, USA) in duplicate. The plates were incubated at $37^{\circ} \mathrm{C}$ for $48 \mathrm{~h}$ and the number of colony forming units $(\mathrm{cfu}) / \mathrm{mL}$ was calculated. Then, 5 colonies representative of the different colonial morphology observed were isolated and stored in gelose agar at room temperature until identification. The strains obtained were identified using the API 20E system (Biolab-Meriéux, France).

The chi-square test was applied to correlate the positive and negative cases with tongue coating area and thickness $(\alpha=0.05)$. Fisher's exact test was used to compare the positive and negative cases with gender, age group, smoking habit, and presence of dentures. Statistically significant difference was considered when $p<0.05$.

\section{RESULTS}

Forty-three percent the individuals showed one or an association of the studied bacterial families (Enterobacteriaceae, Pseudomonadaceae, Moraxellaceae and Xanthomonadaceae) on the tongue dorsum. The isolated presence of Enterobacteriaceae and Pseudomonadaceae was observed among $29 \%$ and $6 \%$ of the volunteers, respectively. Higher prevalence was observed among individuals between 40 and 50 years of age (Table 1) and non-smokers (Table $2)$. No statistically significant $(\mathrm{p}=0.053)$ associations were found between the prevalence of these microorganisms and gender.

Six volunteers wore complete dentures and out of these, 4 presented Enterobacteriaceae and/or Pseudomonadaceae. Nine wore removable partial dentures, and of these 6 were

TABLE 1- Number of volunteers that presented Enterobacteriaceae, Pseudomonadaceae and Moraxellaceae on the tongue dorsum according to the age group

\begin{tabular}{llllll}
\hline Age group & \multicolumn{2}{c}{ Positive Cases } & \multicolumn{2}{c}{ Negative Cases } & \multicolumn{2}{c}{ Total } \\
& $\mathbf{n}$ & $\%$ & $\mathbf{n}$ & & $\mathbf{N}$ \\
\hline $30-39$ & 16 & 37.2 & 40 & 70.17 & 56 \\
$40-50$ & 27 & 62.8 & 17 & 29.83 & 44 \\
\hline
\end{tabular}

Fisher's exact test $(p=0.001)$. 
positive for the studied microorganisms. Fourteen wore fixed dentures, and of these 6 presented Enterobacteriaceae and/ or Pseudomonadaceae. No statistically significant association was found in the prevalence of the studied families and the presence of dentures $(\mathrm{p}=0.890)$.

Combined presence of Enterobacteriaceae and Pseudomonadaceae was observed in $6 \%$ of the sample, and the association of Enterobacteriaceae, Pseudomonadaceae and Moraxellaceae was present in $1 \%$ of the population (data not shown).

A total of 63 isolates were obtained from 43 positive individuals. Enterobacter cloacae was the most prevalent strain $(22.3 \%)$, followed by Pasteurella pneumotropical haemolytica (14.3\%). Of the Pseudomonadaceae family, Chryseomonas luteola was the most prevalent species (9.5\%) (Table 3).

The simultaneous presence of two or more species on the tongue dorsum was observed in 16 volunteers. E. cloacae was the species that appeared more frequently in association with other species, such as Chryseomonas luteola, Pantotea spp., Flavimonas oryzihabitans, Klebsiella pneumoniae, Yersinia enterocolitica, Shigella spp. and Pasteurella pneumotropica. Other combinations included Klebsiella oxytoca and C. luteola, Klebsiella oxytoca and Serratia plymuthica and Shigella spp. and K. pneumoniae rhinoscleromatis.

TABLE 2- Number of volunteers that presented Enterobacteriaceae, Pseudomonadaceae and Moraxellaceae on the tongue dorsum according to the smoking habit

\begin{tabular}{|c|c|c|c|c|c|}
\hline \multirow[t]{2}{*}{ Smoking habit } & \multicolumn{2}{|c|}{ Positive Cases } & \multicolumn{2}{|c|}{ Negative Cases } & \multirow{2}{*}{$\begin{array}{c}\text { Total } \\
\mathbf{N}\end{array}$} \\
\hline & $\mathbf{n}$ & $\%$ & $\mathbf{n}$ & $\%$ & \\
\hline Smoking & 6 & 13.95 & 14 & 24.56 & 20 \\
\hline No smoking & 37 & 86.05 & 43 & 75.44 & 80 \\
\hline
\end{tabular}

Fisher's exact test $(p=0.0485)$

TABLE 3- Frequency of isolation and mean log of colony forming unit per milliliter (cfu/mL) for the Enterobacteriaceae, Pseudomonadaceae, Moraxellaceae and Xanthomonadaceae species on the tongue dorsum of the 100 volunteers

\begin{tabular}{|c|c|c|c|}
\hline \multirow[t]{2}{*}{ Species } & \multicolumn{2}{|c|}{ Frequency of Isolation } & \multirow[t]{2}{*}{ Mean log of cfu/mL } \\
\hline & $\mathbf{n}$ & $\%$ & \\
\hline Enterobacter cloacae & 14 & 22.3 & 2.50 \\
\hline Pasteurella pneumotropica/haemolytica & 9 & 14.3 & 4.14 \\
\hline Pantoea spp. 1 & 6 & 9.5 & 3.18 \\
\hline Chryseomonas luteola & 6 & 9.5 & 3.79 \\
\hline Flavimonas oryzihabitans & 3 & 4.7 & 3.46 \\
\hline Klebsiella oxytoca & 3 & 4.7 & 2.69 \\
\hline Klebsiella pneumoniae rhinoscleromatis & 3 & 4.7 & 3.90 \\
\hline Shigella spp. & 3 & 4.7 & 4.19 \\
\hline Pantoea spp. 2 & 2 & 3.2 & 1.54 \\
\hline Pseudomonas aeruginosa & 2 & 3.2 & 4.93 \\
\hline Yersinia enterocolitica & 2 & 3.2 & 4.08 \\
\hline Klebsiella pneumoniae pneumoniae & 2 & 3.2 & 2.84 \\
\hline Acinetobacter baumannii/calcoaceticus* & 1 & 1.6 & 1.00 \\
\hline Enterobacter cancerogenus & 1 & 1.6 & 2.55 \\
\hline Klebsiella ornithinolytica & 1 & 1.6 & 2.50 \\
\hline Klebsiella pneumoniae ozaenae & 1 & 1.6 & 3.20 \\
\hline Pseudomonas fluorescens/putida & 1 & 1.6 & 2.85 \\
\hline Serratia marcescens & 1 & 1.6 & 1.60 \\
\hline Serratia plymuthica & 1 & 1.6 & 1.60 \\
\hline Stenotrophomonas maltophilia** & 1 & 1.6 & 2.14 \\
\hline Total & 63 & 100 & 2.93 \\
\hline
\end{tabular}

* Moraxellaceae family; ${ }^{* \star X a n t h o m o n a d a c e a e ~ f a m i l y . ~}$ 
TABLE 4- Number of volunteers that presented Enterobacteriaceae, Pseudomonadaceae and Moraxellaceae on the tongue dorsum according to the tongue coating area

\begin{tabular}{lrrrrr}
\hline Area & \multicolumn{2}{c}{ Positive Cases } & \multicolumn{2}{c}{ Negative Cases } & \multicolumn{2}{c}{ Total } \\
& $\mathbf{n}$ & $\mathbf{0}$ & $\mathbf{n}$ & $\mathbf{N}$ & \\
\hline 0 & 7 & 16.28 & 10 & 17.55 & 17 \\
1 & 26 & 60.46 & 38 & 66.66 & 64 \\
2 & 8 & 18.6 & 9 & 15.79 & 17 \\
3 & 2 & 4.66 & 0 & 0 & 2 \\
\hline
\end{tabular}

Chi-square Test $\left(\chi^{2}=2.936\right),(p=0.4016)$.

TABLE 5- Number of volunteers that presented Enterobacteriaceae, Pseudomonadaceae, Moraxellaceae and Xanthomonadaceae on the tongue dorsum according to the tongue coating thickness

\begin{tabular}{lrrrrr}
\hline Thickness & \multicolumn{2}{c}{ Positive Cases } & \multicolumn{2}{c}{ Negative Cases } & \multicolumn{2}{c}{ Total } \\
& $\mathbf{n}$ & $\mathbf{\%}$ & $\mathbf{n}$ & $\mathbf{N}$ & \\
\hline 0 & 7 & 16.28 & 10 & 17.55 & 17 \\
1 & 14 & 32.56 & 15 & 26.32 & 29 \\
2 & 21 & 48.84 & 30 & 52.63 & 51 \\
3 & 1 & 2.32 & 2 & 3.5 & 3 \\
\hline
\end{tabular}

Chi-square test $\left(\chi^{2}=0.536\right),(p=0.911)$.

Regarding tongue coating, a high percentage of subjects with scores 1 for coating area and scores 1 and 2 for thickness was observed (Tables 4 and 5). No significant associations among scores of coating area $(p=0.402)$ or thickness $(p=0.911)$ and detection of microorganisms were found.

\section{DISCUSSION}

In this study, the prevalence of Enterobacteriaceae and/ or Pseudomonadaceae, Moraxellaceae and Xanthomonadaceae in the posterior region of the tongue dorsum was $43 \%$. Studies conducted in Brazil reported similar values, though they analyzed oral rinse samples. Santos, et al. ${ }^{17}$ observed that the prevalence of these microorganisms in the oral cavity of individuals with chronic periodontitis was of $43.18 \%$, and in another study, Santos and Jorge ${ }^{16}$ observed higher prevalence of Enterobacteriaceae and/or Pseudomonadaceae in the oral cavity $(51 \%)$. These results might indicate that, as suggested by previous studies ${ }^{3,25}$, tongue can be the primary reservoir of microorganisms from these families.

Out of the positive volunteers in this study, $69.05 \%$ presented Enterobacteriaceae in posterior dorsum of the tongue. On one hand, there was a significant difference between the prevalence observed in our study and other analyzing the same age group. Sedgley and Samaranayake ${ }^{19}$, in a study conducted in Hong Kong with oral rinse samples (32\%). Goldberg, et al. ${ }^{5}$ analyzing saliva, periodontal pocket and tongue dorsum samples observed a prevalence of Enterobacteriaceae of $27.1 \%$ in patients with halitosis. Also, Leung, et al. ${ }^{8}$, evaluated mouth rinse samples of individuals after radiotherapy treatment at head and neck region, and observed that the prevalence of Enterobacteriaceae in individuals between the ages of 48 and 60 years was $32 \%$. On the other hand, the results of the present study were similar to those obtained by Leung, et al. ${ }^{9}$ for individuals $>$ 60 years of age $(62.5 \%)$. More studies in the age group of $>60$ years in a Brazilian population may clarify these differences.

There was greater prevalence of positive cases for Enterobacteriaceae and Pseudomonadaceae in the age group from 40 to 50 years of age in the present study. Sedgley and Samaranayake $^{18}$ also observed prevalence of Enterobacteriaceae with statistically significant results in the older age groups (over 50 years of age). In a study with volunteers from 43 to 60 years of age and over 60 years of age, Leung, et al. ${ }^{9}$ found higher prevalence of Enterobacteriaceae in the volunteers over the age of 60 years. Further research analyzing other age groups is necessary.

In this study, no statistically significant associations were found between the prevalence of these microorganisms and gender. Similar results have been reported elsewhere ${ }^{16,19}$. On the other hand, higher prevalence of positive cases among males $(\mathrm{p}<0.05)$ was observed in another study ${ }^{17}$.

Previous studies reported the role of complete dentures as a potential reservoir of respiratory pathogens and facilitating factor for the colonization of the oropharyn $\mathrm{x}^{22,23}$, in this study no correlation between the presence of 
microorganisms and dentures could be proven. This result may be related to the small number of dentures users in the sample. Future studies including larger population of denture users could better clarify this correlation.

Enterobacter cloacae was the most frequently isolated Enterobacteriaceae in this study, corroborating the data reported by Sedgley and Samaranayake ${ }^{19}$, Santos and Jorge ${ }^{16}$, Santos, et al. ${ }^{17}$ and Leung, et al. ${ }^{8}$. On the other hand, Galili, et al. ${ }^{4}$, studying leukemia patients, observed that the most isolated Enterobacteriaceae was Klebsiella followed by Enterobacter. The most frequently isolated Pseudomonadaceae species was Chryseomonas luteola, which was found in 9 volunteers, with a prevalence of $9.38 \%$. Chryseomonas luteola was found in 1 patient with a prevalence of $2.08 \%$ in a previous ${ }^{17}$. However, according to Leung, et al. ${ }^{9}$ the most prevalent Pseudomonadaceae in adult oral cavity was Pseudomonas fluorescens/putida (5.3\%) and Stenotrophomonas maltophilia (5.3\%).

The important correlation of $K$. pneumoniae in the oral cavity and risk of aspiration pneumonia in susceptible individuals was cited recently ${ }^{27}$. In the population of stroke sufferers studied by these researchers, this species was the most frequently identified coliform bacteria. In the present study, carried out among volunteers without any systemic disease, this species was observed in small proportion of the population $(3.12 \%)$.

Out of the 100 volunteers analyzed in the present study, the greatest prevalence of tongue coating occurred in the posterior third of the tongue. Similar studies ${ }^{11,13}$, also observed the greatest prevalence of tongue coating in the posterior region of the tongue dorsum. The anterior two thirds of the tongue dorsum are constantly rubbed by the hard palate, possibly cleaning the tongue ${ }^{21}$. The posterior third of the tongue is in contact with the soft palate, where there is no roughness and cleaning effect, and thus it is more prone to accumulate tongue coating. According to Rosenberg ${ }^{15}$, nasal and paranasal secretions may accumulate in the posterior region of the tongue dorsum, favoring the formation of tongue coating.

There was no statistically significant relation between the area and thickness of tongue coating and the presence of Enterobacteriaceae and/or Pseudomonadaceae in this study. Mantilla Gomez, et al. ${ }^{11}$ did not find any significant difference between the coloring and thickness of tongue coating in the dorsal region and bacterial load in saliva samples.

\section{CONCLUSIONS}

According to the data of the present study, it may be concluded that the prevalence of individuals positive for Enterobacteriaceae and/or Pseudomonadaceae in the posterior region of the tongue dorsum was high, especially among volunteers in the 40-50-year-old age group and nonsmokers. There was no significant correlation between the presence of Enterobacteriaceae and Pseudomonadaceae and tongue coating, gender or denture use.

\section{REFERENCES}

1- Arora HK, Chapman GB. Transmission electron microscope study of bacterial morphotypes on the anterior dorsal surface of human tongues. Anat Rec. 2000;259:276-87.

2- Baydas B, Uslu H, Yavuz I, Ceylan I, Dagsuyu IM. Effect of chronic nail-biting on the oral carriage of Enterobacteriaceae. Oral Microbiol Immunol. 2007;22:1-4.

3- Faveri M, Feres M, Shibli JA, Hayacibara RF, Hayacibara MM, Figueiredo LC. Microbiota of the dorsum of the tongue after plaque accumulation: an experimental study in humans. J Periodontol. 2006;77:1539-46

4- Galileu D, Donitza A, Garfunkel A, Sela MN. Gram-negative enteric bacteria in the oral cavity of leukemia patients. Oral Surg Oral Med Oral Pathol. 1992;74:459-62.

5- Goldberg S, Cardash H, Browining H, Sahly H, Rosenberg M. Isolation of Enterobacteriaceae from the mouth and potential association with malodor. J Dent Res. 1997;76:1770-5.

6- Hägg U, Kaveewatcharanont P, Samaranayake YH, Samaranayake LP. The effect of fixed orthodontic appliances on the oral carriage of Candida species and Enterobacteriaceae. Eur J Orthod. 2004;26(6):623-9.

7- Kazor CE, Mitchell PM, Lee AM, Stokes LN, Loesche WJ, Dewhirst FE, et al. Diversity of bacterial populations on the tongue dorsa of patients with halitosis and healthy patients. J Clin Microbiol. 2001;41:558-63.

8- Leung WK, Jin LJ, Yam WC, Samaranayake LP. Oral colonization of aerobic and facultatively anaerobic gram-negative rods and cocci in irradiated, dentate, xerostomic individuals. Oral Microbiol Immunol. 2001;16:1-9.

9- Leung WK, Yau JY, Cheung BP, Jin LJ, Zee KY, Lo EC, et al. Oral colonisation by aerobic and facultatively anaerobic Gram-negative rods and yeast in Tibetans living in Lhasa. Arch Oral Biol. 2003;48:117-23.

10- Mager DL, Ximenez-Fyvie LA, Haffajee AD, Socransky SS. Distribution of selected bacterial species on intraoral surfaces. J Clin Periodontol. 2003;30:644-54.

11- Mantilla Gomez S, Danser MM, Sipos PM, Rowshani B, van der Velden U, van der Weijden GA. Tongue coating and salivary bacterial counts in healthy/gingivitis subjects and periodontitis patients. J Clin Periodontol. 2001;28:970-8.

12- Napeñas JJ, Brennan MT, Bahrani-Mougeot FK, Fox PC, Lockhart $\mathrm{PB}$. Relationship between mucositis and changes in oral microflora during cancer chemotherapy. Oral Surg Oral Med Oral Pathol Oral Radiol Endod. 2007;103(1):48-59.

13- Quirynen M, Avontroodt P, Soers C, Zhao H, Pauwels M, van Steenberghe D. Impact of tongue cleansers on microbial load and taste. J Clin Periodontol. 2004;31:506-10.

14- Roldán S, Herrera D, Sanz M. Biofilms and the tongue: therapeutical approaches for the control of halitosis. Clin Oral Investig. 2003;7:18997.

15- Rosemberg M. Clinical assessment of bad breath: current concepts. J Am Dent Assoc. 1996;4:475-82.

16- Santos SSF, Jorge AOC. Presença de Enterobacteriaceae e Pseudomonadaceae na cavidade bucal humana. Rev Odontol UNESP. $1998 ; 27: 473-84$.

17- Santos SSF, Loberto JCS, Martins CAP, Jorge AOC. Prevalência e sensibilidade in vitro de Enterobacteriaceae e Pseudomonas isoladas da cavidade bucal e bolsa periodontal de pacientes com periodontite crônica. PGRO-Pos Grad Rev Odontol. 2002,5:74-83. 
18- Sedgley CM, Samaranayake LP. Oral and oropharyngeal prevalence of Enterobacteriaceae in humans: a review. J Oral Pathol Med. 1994;23:104-13.

19- Sedgley CM, Samaranayake LP. The oral prevalence of aerobic and facultatively anaerobic Gram-negative rods and yeasts in Hong Kong Chinese. Arch Oral Biol. 1994;39:459-66.

20- Slots J, Feik D, Rams TE. Prevalence and antimicrobial susceptibility of Enterobacteriaceae, Pseudomonadaceae and Acinetobacter in human periodontis. Oral Microbiol Immunol. 1990;5:149-54.

21- Spielman AI, Bivona P, Rifkin BR. Halitosis a common oral problem. N Y State Dent J. 1996;63:36-42.

22- Sumi Y, Miura H, Sunakawa M, Michiwaki Y, Sakagami N. Colonization of denture plaque by respiratory pathogens in dependent elderly. Gerodontology. 2002;19:25-9.

23- Sumi Y, Kogami H, Ohtsuka Y, Kakinoki Y, Haruguchi Y, Miyamoto $\mathrm{H}$. High correlation between the bacterial species in denture plaque and pharyngeal microflora. Gerodontology. 2003;20:84-7.

24- Tanaka M, Yamamoto Y, Kuboniwa M, Nonaka A, Nishida N, Maeda $\mathrm{K}$, et al. Contribution of periodontal pathogens on tongue dorsa analyzed with real-time PCR to oral malodor. Microbes Infect. 2004;6:1078-83.

25- Tanner AC, Milgrom PM, Kent R Jr, Mokeem SA, Page RC, Riedy $\mathrm{CA}$, et al. The microbiota of young children from tooth and tongue samples. J Dent Res. 2002;81(10):53-7.

26- Tsang CS, Samaranayake LP. Oral yeasts and coliforms in HIV-infected individuals in Hong Kong. Mycoses. 2000;43(7-8):303-8.

27- Zhu HW, McMillan AS, Mc Grath C, Li LS, Samaranayake LP. Oral carriage of yeasts and coliforms in stroke sufferers: a prospective longitudinal study. Oral Dis. 2008;14(1):60-6. 